\title{
In Vivo
}

National Cancer Institute

\section{Source}

National Cancer Institute. In Vivo. NCI Thesaurus. Code C15744.

Located or occurring in the body. 